\title{
Neues von der Palmengarten-Gesellschaft „Freunde des Palmengartens e. V.“
}

\section{Die Gesellschaft „Freunde des Palmengartens“ kann erneut auf ein weiteres erfolgreiches Jahr zurückblicken. Die Aufgaben der Gesellschaft bestehen darin, den Palmengarten zu fördern. Im Jahr 2009 wurden folgende Projekte unterstïtzt:}

Restfinanzierung der Felswand mit Wasserfall im Savannenhaus des Tropicariums: $€ 35000$. Leider verzögert sich die Inbetriebnahme des Wasserfalls wegen technischer Probleme und der Insolvenz der ausführenden Firma. Die endgültige Bepflanzung des Gewächshauses mit speziellen australischen Pflanzen wird sich deshalb noch etwas herauszögern.

Mit $€ 19000$ wurde der Druck der Palmengarten-Programme unterstützt. Da auf jedem Flyer auf die finanzielle Unterstützung durch die Gesellschaft hingewiesen wird, tritt ein beachtlicher Werbe-Effekt für die Gesellschaft auf. Diese Finanzierung ist auch für 2010 und folgende Jahre geplant.

Referentenhonorare in Höhe von insgesamt $€ 12000$ für Veranstaltungen des Palmengartens (Vorträge, Führungen, Praxis-Seminare und Schüler-Seminar) konnten ebenfalls von der Gesellschaft übernommen werden.

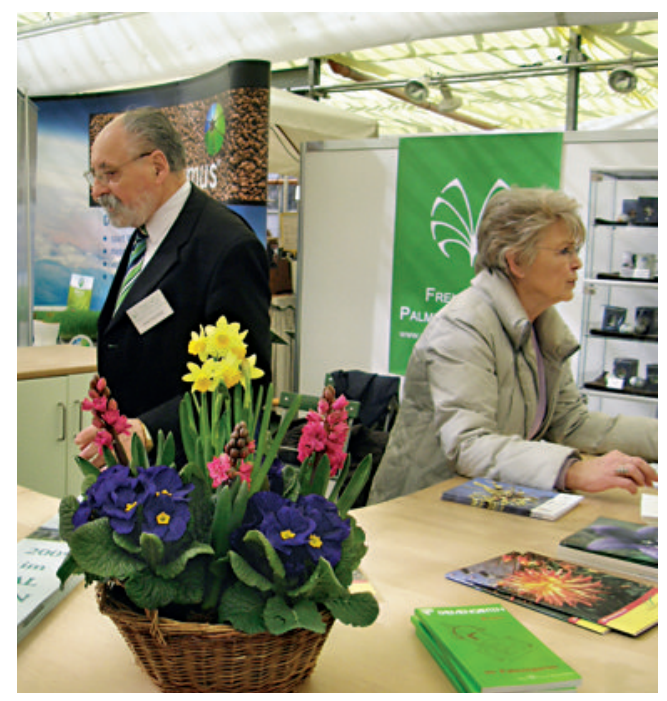

Es wurden Bilderrahmen für Ausstellungen des Palmengartens im Wert von $€ 3500$ angeschafft.

Außerdem wurde eine Vitrine des Palmengartens im Westin-Hotel (ehemals Arabella), mit $€ 3000$ finanziert:

Ehrenamtliche Helferinnen und Helfer übernahmen die Aufsicht während der Rosenausstellung.

Verkauf für den Palmengarten von Palmengarten-Tassen aus Höchster Porzellan, GinkgoAnstecknadeln etc. durch den Helferkreis.

In diesem Jahr werden wiederum der Druck der Palmengartenprogramme und die Referentenhonorare finanziert, außerdem ein Praktikantenplatz für 12 Monate. Aufsicht bei den Ausstellungen des Palmengartens erfolgt dieses Jahr über mehr als drei Monate hinweg vom 20. Mai bis 5. September 2010, und zwar bei den Ausstellungen "StadtGrün" und „GOETHE und die Pflanzenwelt"; wenn Sie noch mitmachen möchten, melden Sie sich bitte bei der Geschäftsstelle.

Und natürlich unterstützt der Helferkreis den Palmengarten auch dieses Jahr wieder, insbesondere durch den Verkauf für den Palmengarten von Palmengarten-Tassen und Ginkgo-Blättern.

\section{Auch für die Mitglieder selbst gab es Highlights:}

Tagesfahrten nach Sangerhausen (37 Teilnehmende) und Umgebung sowie nach Mannheim in den Luisenpark (52 Teilnehmende).

Abb. 1: DORRIT RÖHNERT und HERBERT BiLlensteINER am Stand der Freunde des Palmengartens während der Gartenmesse 2009. 


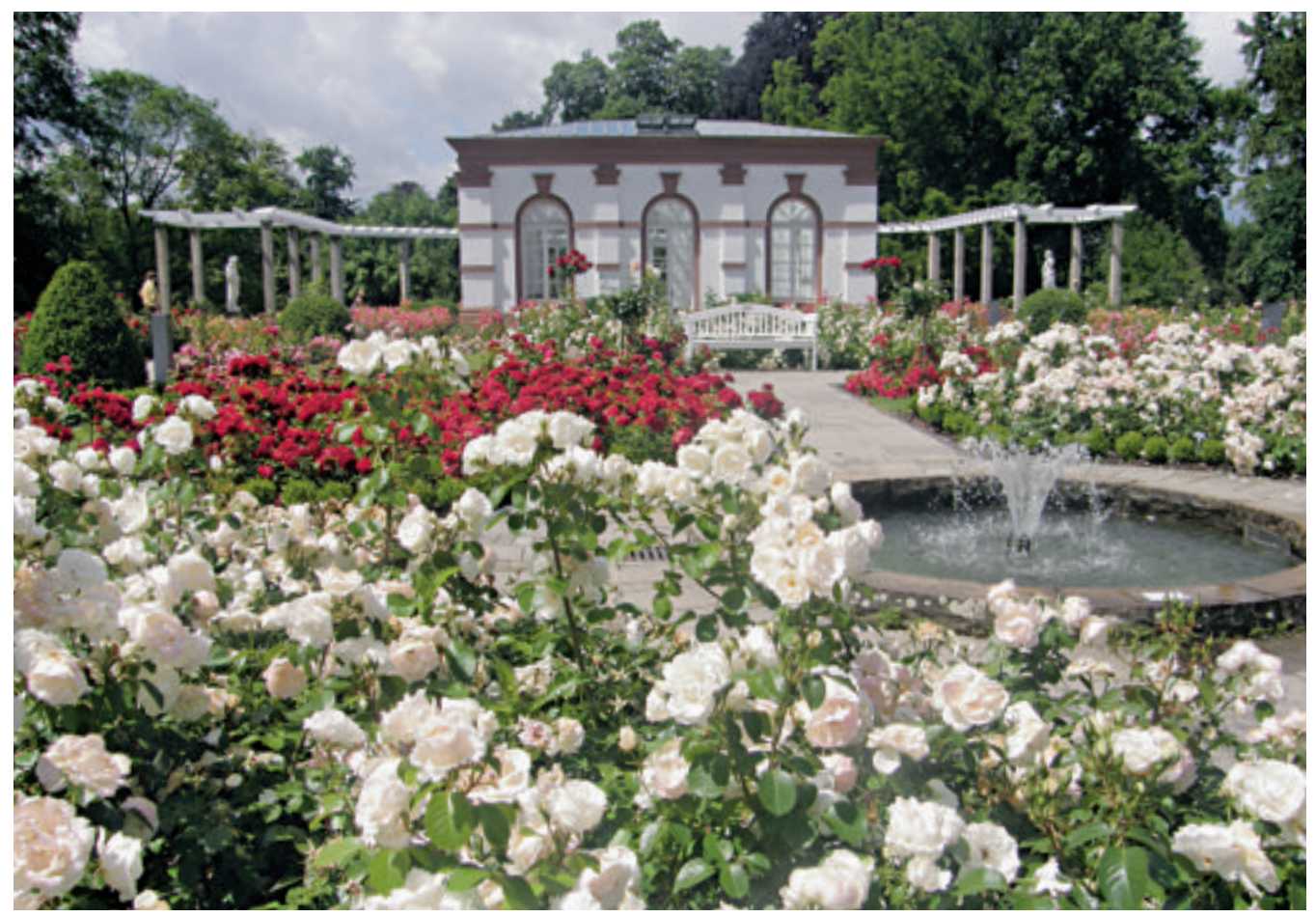

Mehrtagesfahrt nach Venetien (34 Teilnehmende); wegen der großen Nachfrage wurde die Fahrt sogar wiederholt (18 Teilnehmende; zur Venetien-Fahrt vgl. separaten Bericht).

Weihnachtstreffen im Großen Festsaal des Casinos des Westend-Campus. An der Veranstaltung nahmen über 250 Mitglieder teil. Der Große Festsaal war ein würdiger Rahmen, für die meisten Teilnehmer war es das erste Mal, dass sie diesen denkmalgeschützten Repräsentationsraum des Poelzig-Baus erleben konnten. Es gab eine musikalische Begleitung durch das Ehrenmitglied HeINZ MARX und sein Salon-Orchester Allegro sowie ein Kinderprogramm durch die Grüne Schule.

In diesem Jahr fand die Mehrtagesfahrt nach Madeira bereits statt (12. bis 19. April 2010, 17 Teilnehmende). Die Tagesfahrten führen dieses Jahr nach Bad Nauheim zur Landesgartenschau (29. Mai 2010) und nach Schlüchtern zum Schloss Rambach (10. Juli 2010).

Abb. 2: Nach der Neugestaltug hat sich der Rosengarten sehr gut entwickelt, hier ein Eindruck von Juni 2009.
Außerdem findet in diesem Jahr wieder ein Sommertreffen der Mitglieder im Palmengarten statt, und zwar am Sonntag, den 13. Juni 2010 während der Rosenausstellung. Die Einladung erfolgte bereits schriftlich im März zusammen mit der Einladung zur diesjährigen Jahresmitgliederversammlung am 21. April 2010. Das Sommertreffen bietet wieder eine gute Gelegenheit, Kontakte zu anderen Mitgliedern zu knüpfen und Präsenz der Gesellschaft im Palmengarten zu demonstrieren; Kinder sind wieder ausdrücklich willkommen, sie können am Programm der Grünen Schule teilnehmen. Eine Anmeldung ist für das Sommertreffen nicht erforderlich, aber bitte lassen Sie sich am Stand der Gesellschaft registrieren.

\section{Unseren Mitgliedern und dem Palmengarten wünschen wir ein weiteres erfolgreiches Jahr.}

Im April 2010

Klaus Urban, Vorsitzender 